\title{
The Appearance of Ferrielectric Phases in Confined Liquid Crystal Studied by Photon Correlation Spectroscopy
}

\author{
Yuri Panarin \\ Technological University Dublin, yuri.panarin@tudublin.ie \\ Fouad M. Aliev \\ University of Puerto Rico \\ Charles Rosenblatt \\ Case Western Reserve University
}

Follow this and additional works at: https://arrow.tudublin.ie/engscheleart2

Part of the Electrical and Computer Engineering Commons

\section{Recommended Citation \\ Panarin, Y.P. (1999). The appearance of ferrielectric phases in confined liquid crystal studied by photon correlation spectroscopy. Molecular Crystals and Liquid Crystals vol. 328, pp. 93-100}

This Article is brought to you for free and open access by the School of Electrical and Electronic Engineering at ARROW@TU Dublin. It has been accepted for inclusion in Articles by an authorized administrator of ARROW@TU Dublin. For more information, please contact arrow.admin@tudublin.ie, aisling.coyne@tudublin.ie, gerard.connolly@tudublin.ie.

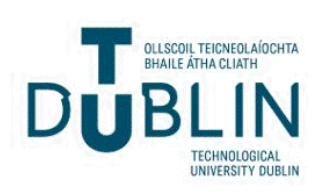




\title{
The Appearance of Ferrielectric Phases in Confined Liquid Crystal Studied by Photon Correlation Spectroscopy
}

\author{
YURI P. PANARIN ${ }^{\mathrm{a}}$, FOUAD M. ALIEV ${ }^{\mathrm{a}}$ and \\ CHARLES ROSENBLATT ${ }^{\mathrm{b}}$ \\ ${ }^{a}$ Department of Physics, University of Puerto Rico, P.O. Box 23343, PR, USA; and \\ ${ }^{\mathrm{b}}$ Department of Physics, Case Western Reserve University, Cleveland, $\mathrm{OH}$, USA
}

The influence of confinement on the dynamic properties and appearance of ferrielectric phases has been investigated by dynamic light scattering, performed in the bulk AFLC as well as confined in $200 \AA$ and $2000 \AA$ cylindrical pores. The analysis of the experimental results shows that the ferrielectric phases are not formed in $200 \AA$ pores, while they appear in $2000 \AA$ A pores as well as in bulk.

Keywords: antiferroelectric liquid crystal; photon correlation spectroscopy

\section{INTRODUCTION}

The appearance of ferrielectric phases in antiferroelectric liquid crystals (AFLC) can be understood as a result of the competition between the antiferro- and ferroelectric interactions in adjacent smectic layers that stabilize the $\mathrm{SmC}_{\mathrm{A}}$ and the $\mathrm{SmC}^{*}$ phases. In ferrielectric liquid crystals the directions of the local spontaneous polarizations in any two neighboring smectic layers can be ferroelectric-like or antiferroelectric-like. The antiferroelectric order is believed to exist due to steric factors since it appears in racemate chiral liquid 
crystals $^{[1]}$ as well as in non-chiral tilted smectics ${ }^{[2]}$. On the other hand we are unaware of any report of ferrielectricity in non-chiral and/or racemic liquid crystals. It seems, therefore, that the existence of local spontaneous polarization is a determining factor for ferrielectricity. The Ising model ${ }^{[3]}$, which takes into account the long-range electrostatic interaction between smectic layers, predicts the existence of an infinite number of ferrielectric phases $^{[i]}$. According to the Ising model, these phases can be characterized by the parameter $q_{T}=F /(A+F)$, which denotes the fraction of ferroelectric ordering $(F)$ which appears together with antiferroelectric ordering $(A)$ in the periodic structure. Following this definition, the $\mathrm{SmC}_{A}$ is the antiferroelectric phase with $q_{T}=0$, the $\mathrm{SmC}_{\gamma}$ is ferrielectric phase with $q_{T}=1 / 3$, the $\mathrm{AF}$ is antiferroelectric phase with $q_{T}=1 / 2$, and the $\mathrm{SmC}^{*}$ is ferroelectric with $q_{T}=1$. Additionally, other ferrielectric subphases (FiLC, $\mathrm{SmC}_{\alpha}$ ) have been found, which cannot be described by the $q_{T}$ parameter.

The Ising model was developed for bulk liquid crystals; therefore the question "What happens to ferrielectric phases in confined liquid crystals?" is of great interest and importance for understanding the nature of ferrielectricity in liquid crystals. In parallel cylindrical pores the molecular tilt directions are equivalent with respect to azimuthal angle (if planar boundary conditions are assumed). Therefore in cylindrical pores the effect of the surface interaction can be ignored and we can investigate the effect of confinement on the appearance of ferrielectric phases.

Dynamic properties of bulk antiferroelectric and ferrielectric phases have been investigated by dielectric ${ }^{[4-10]}$, electrooptic ${ }^{[5,10]}$ and photon correlation ${ }^{[11}$. 16) spectroscopy. Summarizing the results of these investigations, two collective relaxation processes have been observed in antiferroelectric phase: 
(i) antiphase mode ${ }^{[7]}$ (or optic-like phason ${ }^{[15]}$ ) and (ii) in-phase mode ${ }^{[7]}$ (or sound-like phason ${ }^{[14]}$ ).

Investigation of AFLC confined in cylindrical pores is rather difficult, because of specific confining geometry. In dielectric spectroscopy experiment, applied to our system the direction of electric field is along the smectic layer normal and the information about collective modes is missed. Polarizing microscopy is not effective because the pore diameter is smaller than light wavelength. Therefore, in this paper we report on the influence of confinement on the appearance of antiferroelectric/ ferrielectric phases in AFLC investigated by photon correlation spectroscopy.

Dynamic light scattering is a powerful technique to investigate dynamic properties of liquid crystals both in bulk and confined geometry. In dynamic light scattering the intensity/intensity autocorrelation function provides information about relaxation processes in the sample, which are different in different ferrielectric/ antiferroelectric phases.

\section{EXPERIMENTAL}

We have performed investigations in bulk AFLC and confined in cylindrical pores of Anopore membranes with different diameters: $200 \AA$ and $2000 \AA$. The AFLC used in the experiments was (R) - 1 - methylheptyl - 4 -(4"- ndodecyloxybiphenyl-4'-carbonoyloxy) - 3 - fluorobenzoate (AS-573, Hull, UK).

This AFLC is of great interest because it possesses a wide variety of different antiferroelectric and ferrielectric. The phase transition sequence of the bulk sample has been determined by the macroscopic polarization measurements and dielectric spectroscopy ${ }^{[9]}$ :

$$
\mathrm{SmC}_{\mathrm{A}} 78 \mathrm{SmC}_{\gamma} 83 \text { AF } 85 \text { FiLC } 91 \text { SmC* } 93 \mathrm{SmA} 106 \text { Is }
$$


In our experiments the bulk liquid crystal was placed between two untreated glass plates having a $100 \mu \mathrm{m}$ gap. The texture of liquid crystal was observed to be planar disordered with a helix. The helical pitch was about $0.2-$ $0.3 \mu \mathrm{m}$ (less than a wavelength of visible light $\lambda$ ). For the investigations in the confined geometry we used Anopore membranes of thickness $60 \mu \mathrm{m}$. The liquid crystal structure within the pores is believed to be helical, with helical axes along the pores, because a selective reflection in blue region was observed.

We performed photon correlation spectroscopy using a $\mathrm{He}-\mathrm{Ne}$ laser operating at wavelength 6328A, and an ALV-5000/Fast Digital Multiple Tau Correlator (real time) operating over delay times from $12.5 \mathrm{~ns}$ up to $10^{3} \mathrm{~s}$. The depolarized component of scattered light was investigated. All the data discussed below were obtained at $\theta=30^{\circ}$. The data were taken after stabilizing the temperature for one hour at each value of $T$, sufficiently long to guarantee thermal equilibrium.

\section{RESULTS}

The temperature-dependence measurements were carried out in the temperature range $72{ }^{\circ} \mathrm{C}<\mathrm{T}<94{ }^{\circ} \mathrm{C}$. Figure $1(\mathrm{a}-\mathrm{c})$ presents the intensity/ intensity autocorrelation functions of AS573 in the $100 \mu \mathrm{m}$ cell (bulk) and in pores for three different temperatures corresponding to different phases over the time range $10^{-2}<\tau<10^{4} \mathrm{~ms}$.

The autocorrelation functions of the bulk AFLC consist of (at most) three decays corresponding to three relaxation processes (Fig. Ib, curve 1): (i) the "fast" process ( $\tau=27 \mu \mathrm{s}$ ), (ii) the "middle frequency" process ( $\tau=50 \mathrm{~ms}$ ) and (iii) the "low frequency" process ( $\tau=850 \mathrm{~ms}$ ). From these figures we observe 
some similarities and differences in the shape of autocorrelation functions obtained in bulk and in confined liquid crystal.

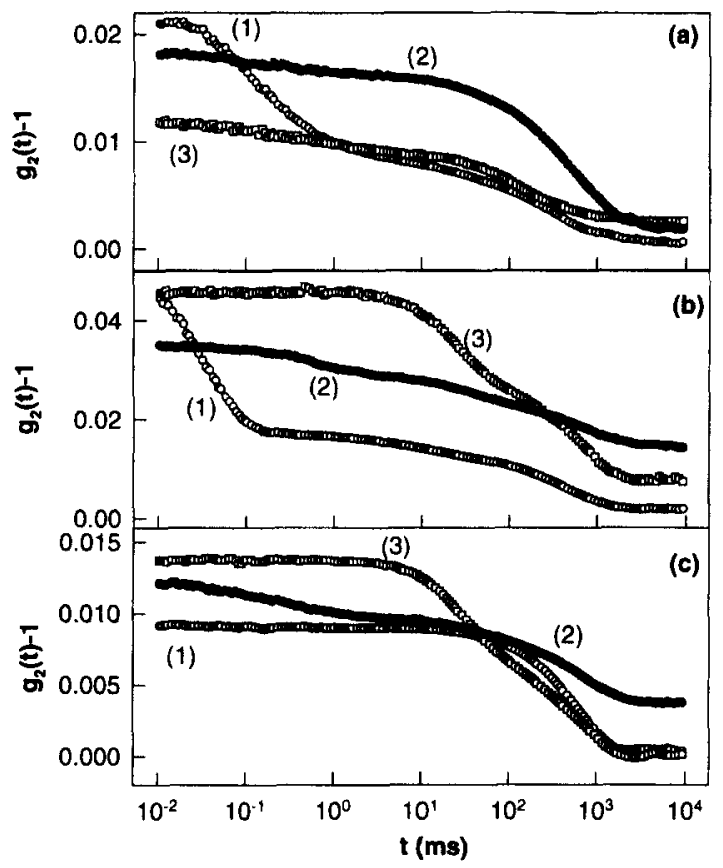

FIGURE 1 Intensity/intensity autocorrelation functions of AS573 in bulk (1), in $2000 \AA$ pores (2), and in $200 \AA$ pores (3) measured at (a) $75.5^{\circ} \mathrm{C}$, (b) $85.5^{\circ} \mathrm{C}$, and (c) $91.5^{\circ} \mathrm{C}$.

The amplitude of the fast relaxation process strongly depends on the temperature. In the bulk sample this process is very prominent in the temperature range below $88^{\circ} \mathrm{C}$, which corresponds to antiferroelectric/ ferrielectric phases (Fig. la,b, curve 1). In the temperature region above $88^{\circ} \mathrm{C}$ the contribution of the fast relaxation process decreases with increasing 
temperature, and it vanishes in $\mathrm{SmC}^{*}$ phase (Fig.1c, curve 1). This fact allows us to assign the fast relaxation process to fluctuations of the antiferroelectric order parameter ${ }^{[7,10]}$ (or optic-like phason ${ }^{[15]}$ ).

Two low frequency relaxation processes, which appear in all smectic phases, including the $\operatorname{SmA}$, have been assigned to hydrodynamic fluctuation modes due to the existence of one-dimensional modulation of smectic layers (stripe-domains) $^{[17]}$.

The fast relaxation process exists in the temperature range corresponding to antiferroelectric/ ferrielectric phases in bulk as well as in $2000 \AA$ pores. Therefore one can conclude that the antiferroelectric/ ferrielectric phases appear in $2000 \AA$ pores as well as in bulk.

In $200 \AA$ pores the fast relaxation process appears only in the $S m C_{A}$ phase. This means that the ferrielectric phases do not appear in $200 \AA$ pores. Combining these facts we can conclude that antiferroelectric order in $200 \AA$ pores does not appear, and the ferrielectric phases are not formed. The reasons for that will be discussed bellow.

Additionally, the analysis of the temperature dependencies of relaxation times shows ${ }^{[16]}$ that the temperatures of the $\mathrm{SmC}_{\mathrm{A}}-\mathrm{SmC}_{\gamma}$ phase transition in bulk and in pores are of comparable value, whereas the temperature of the $\mathrm{SmC}^{*}-\mathrm{SmA}$ phase transition in $2000 \AA$ pores is about $3^{\circ} \mathrm{C}$ lower and in 200 $\AA$ porous sample is about $4^{\circ} \mathrm{C}$ lower than in the bulk sample.

\section{DISCUSSION}

The ferrielectric phases exist in $2000 \AA$ pores and do not exist in 200 $\AA$ pores. This can be understood on the basis of the Ising model, which uses a free energy of the form ${ }^{[3]}$ : 


$$
F=-\sum_{i} H S_{i}+\frac{1}{2} \sum_{i, j} J(i-j)\left(\frac{S_{i}+1}{2}\right)\left(\frac{S_{j}+1}{2}\right)
$$

where the spin $S_{i}=1(-1)$ applies to ferroelectric (antiferroelectric) type ordering between two neighboring smectic layers.

According to this model the existence of ferrielectric phases is explained by long range electrostatic interactions between local spontaneous polarizations (spins) of smectic layers, while the antiferroelectric and ferroelectric phases is accounted for by the first term of the equation. The stability of the ferrielectric phases depends on the strength of the potential $J(i-j)$, i.e., how rapidly it varies with the distance between smectic layers. In the bulk liquid crystal the smectic layer thickness $(L \approx 30 \AA)$ is much smaller than the dimensions of the area of smectic layers, giving an interaction along an infinite number of infinite (in extent) smectic layers. In sufficiently small cylindrical pores $(200 \AA)$, the area of the smectic layer becomes commensurate with the smectic layer thickness, the electric field of the spontaneous polarization of smectic layers decays more rapidly (as function of distance) than in bulk and in $2000 \AA$ pores limiting the number of interacting smectic layers. In other words, the shrinking of the area of smectic layers shortens the electrostatic interaction between local spontaneous polarizations in the direction of smectic layer normal. In this case (for $200 \AA$ pores) the electrostatic interactions are not strong enough to form ferrielectric phases.

The observed behavior in cylindrical pores is extremely rich, and provides an important platform for better understanding the nature of ferrielectric phases in antiferroelectric liquid crystalline materials. 


\section{Acknowledgements}

We thank Prof. J. W. Goodby of University of Hull (UK) for providing AFLC material. This work was supported by U.S. Air Force grant F49620-95-10520, DOE grant DE-FG02-94ER75764, and by the National Science Foundations under grants OSR-9452893 and DMR-9502825.

\section{References}

[1] A. Fukuda, Yo. Takanishi, T. Isozaki, K. Ishikawa, and H. Takezoe, J. Mater Chem., 4, 997 (1994).

[2] I. Nishiyama, and J.W. Goodby, J. Mater. Chem., 2, 1015 (1992).

[3] P. Bak, and R. Bruinsma, Phys. Rev. B, 21, 5297 (1980).

[4] K. Hiraoka, A. Taguchi, Yu. Ouchi, H. Takezoe, A. Fukuda, J. J. Appl. Phys., 29, L103 (1990).

[5] K. Hiraoka, H. Takezoe, A. Fukuda, Ferroelectrics, 147, 13 (1993).

[6] S. Hiller, S.A. Pikin, W. Haase, J.W. Goodby, Isa Nishiyama, Jap. J. Appl. Phys., 33, L1096 (1994).

[7] M. Buivydas, F. Gouda, S.T. Lagerwall, and B. Stebler, Liq. Cryst., 18, 879 (1995).

[8] S. Merino, M.R. de la Fuente, Y. Gonzalez, M.A. Perez Jubindo, J.A. Puertolas, Phys. Rev. E, 54, 5169 (1996).

[9] Yu.P. Panarin, O.E. Kalinovskaya, and J.K. Vij, Phys. Rev. E, 55, 2354 (1997).

[10] Yu. P.Panarin, O.E. Kalinovskaya and J.K. Vij, Appl. Phys. Lett., 72,1667 (1998).

[11] H. Sun, H. Orihara, Yo. Ishibashi, J. Phys. Soc. Jap., 60, 4175 (1991).

[12] H. Sun, H. Orihara, Yo. Ishibashi, J. Phys. Soc. Jap. 62, 2066-2073 (1993).

[13] K. Miyachi, M. Kabe, K. Ishikawa, H. Takezoe, A. Fukuda, Ferroelectrics, 147, 147 (1993).

[14] I. Musevic, R. Blinc, B. Zeks, M. Copic, M.M. Witterbrood, Th. Rasing, H. Orihara, Yo. Ishibashi, Phys. Rev. Lett. 71, 1180 (1993).

[15] I. Musevic, A. Rastegar, M. Cepic, B. Zeks, M. Copic, D. Moro, G. Heppke, Phys. Rev. Lett. 77, 1769 (1996).

[16] Yu.P. Panarin, C. Rosenblatt, and F.M. Aliev, Phys. Rev. Lett., 812699 (1998).

[17] A. Tang, and S. Sprunt, Phys. Rev. E., 57, 3050 (1998). 